RAPER, K. B. (1956). J. gen. Microbiol. 14, 716-732

\title{
Dictyostelium polycephalum n.sp.: a New Cellular Slime Mould with Coremiform Fructifications
}

\author{
BY K. B. RAPER \\ Departments of Bacteriology and Botany, University of Wisconsin, Madison, \\ Wisconsin, U.S.A.
}

SUMMARY: A unique species of Dictyostelium is described for which the binomial Dictyostelium polycephalum is proposed because of the seemingly branched character of its fructifications. This slime mould has been isolated repeatedly from samples of surface soil and decomposing leaves collected from deciduous forests in various parts of the United States. As in other members of the Acrasieae, its vegetative phase consists in the independent growth of free-living myxamoebae which feed upon bacterial cells, and its fruiting phase is characterized by the inflowing of these cells to form multicellular organizations preparatory to fructification. D. polycephalum differs from previously known species of the genus, particularly for its formation of cell aggregates which give rise to varying numbers of long, thin migrating pseudoplasmodia, and for the potential capacity of each of these to form subsequently a small coremiform fructification consisting of multiple sorocarps. Whereas some simple sorocarps are regularly produced, fructifications under optimal conditions typically consist of from two to ten adherent sorocarps. In such coremiform structures, the sorophores of individual sorocarps, although clearly distinguishable microscopically, are tightly appressed for approximately three-quarters of their length, at which level they diverge sharply and at their apices bear globose spore heads, or sori. The spores are elliptical to reniforrn as in most species, but germinate by the swelling and dissolution of the spore wall in a median plane rather than by longitudinal splitting.

The genus Dictyostelium, established by Brefeld with his description of D. mucoroides in 1869 , is characterized by simple or irregularly branched fruiting structures, or sorocarps. As in all of the simple cellular slime moulds of the class Acrasieae, the vegetative stage consists in the growth and multiplication of small free-living amoeboid cells, or myxamoebae. In contrast, the fruiting stage is characterized by the aggregation of these myxamoebae into multicellular organizations, termed pseudoplasmodia, followed by their progressive integration and differentiation to form either structural elements of the sterile supportive stalk, or sorophore, or reproductive cells, spores, which with enveloping slime comprise the spore head, or sorus.

Species of Dictyostelium are widely distributed in nature and occur with generally unappreciated frequency in persistent accumulations of well-rotted leaf mould in deciduous forests. Less than a dozen species have been described. Of these, only six are in any sense well known: $D$. mucoroides, very abundant and very variable, is characterized by milk-white sori and generally unbranched, or sparsely branched, sorophores. D. purpureum Olive (1901, 1902), less commonly encountered, differs from the preceding primarily in producing deep purple sori. D. minutum Raper $(\mathbf{1 9 4 1} b)$ represents a consistently diminutive form, but otherwise resembles $D$. mucoroides. D. lacteum Van Tieghem (1880), relatively rare and only recently rediscovered (Raper, 1951), is an 
extremely delicate species characterized by spherical spores. D. giganteum Singh (1947) is a robust species doubtfully separable from $D$. mucoroides. D. discoideum Raper (1935) is characterized by migrating pseudoplasmodia and by erect tapering sorophores that arise from flattened basal disks.

A cellular slime mould differing markedly from any of the above and from any previously described form has been isolated repeatedly during the past five years, and hence merits recognition as a new species. It typically exhibits a migrating pseudoplasmodial stage, thus suggesting Dictyostelium discoideum. Aside from this single character, however, it bears little resemblance to that species. It is distinguished particularly by its coremiform fruiting structures which consist of a variable number of sorocarps. In such compound structures the individual sorophores are easily discernible, these being parallel and closely adherent throughout approximately three-quarters of their total length, then strongly divergent with each terminated by a small globose sorus. Considered individually, the clustered sorocarps would seem to represent a diminutive and simple Dictyostelium; viewed under low magnifications, the coremiform clusters appear as single, well-proportioned structures sometimes bearing as many as $\mathbf{8}$ to 10 spore heads. Because of these equally important characteristics, the species is placed in the genus Dictyostelium and the binomial $D$. polycephalum is proposed for it. A technical description of the newly discovered slime mould, together with brief accounts of its isolation, cultivation, and the more salient features of its developmental history follows:

\section{TECHNICAL DESCRIPTION}

\section{Dictyostelium polycephalum sp.nov.}

Cultum ad 25-30 $\mathrm{C}$ in substrato solido (agar-agar), dilutam faeni infusionem vel dictam 'glucose + peptone' solutionem continente, cum diversis bacteriis fungisque mixtum, praecipue cum Aerobacter aerogenes et Dematium nigrum. Sorocarpi plerumque 375-650 $\mu$. alti, seape singuli sed typice plures usque ad 10 in coremiformen fructificationem fasciculati. Sorophori per majorem longitudinis partem inter se conjuncti, sed apices versus disjuncti. Sori albi vel hyalini, globosi, 45-90 $\mu$. diam. Sporae ellipiticae vel reniformes, plerumque 6.0-7.5 $\mu$. longae, 3.0-3.5 $\mu$. latae.

Hab. Ex foliis putrescentibus et summo solo in deciduis silvis isolatum, South Carolina, Texas, Michigan, Wisconsin et Illinois, U.S.A. (Strain S-4, Maio, 1951 isolatum typus).

Cultures grown upon dilute hay-infusion or dilute glucose + peptone agars in association with a mixed bacterial and fungus flora at temperatures between $\mathbf{2 5}$ and $\mathbf{3 0}^{\circ}$; sorocarps small, often simple but typically clustered to form coremiform fructifications consisting of variable numbers of sorocarps, commonly 375-650 $\mu$. in height, with sorophores appressed throughout most of their length, diverging terminally to bear white to colourless, globose sori $45-90 \mu$. in diameter; spores elliptical to reniform, usually $6 \cdot 0-7 \cdot 5 \times 3.0-3.5 \mu$; myxamoebae form aggregates typical of the group, but these give rise to several migrating pseudoplasmodia which in turn may produce either simple sorocarps or compound fructifications consisting of several sorocarps.

Isolated from decaying leaves and surface soil from deciduous forests, South Carolina, Texas, Michigan, Wisconsin and Illinois, U.S.A. 


\section{ISOLATION AND CULTIVATION}

The type culture of Dictyostelium polycephalum, strain no. S-4, was isolated in May 1951 by the writer and Miss Dorothy Fennell at the Northern Regional Research Laboratory, Peoria, Illinois, U.S.A., from a sample of well-rotted leaf mould collected from a predominantly hardwood forest near Holly Hill, South Carolina. The principal vegetation at the site of the collection consisted of live oaks, maples, sweet gum, and a variety of other deciduous trees and shrubs. The slime mould was isolated in the conventional way. A small portion of the sample $(c .1 .0 \mathrm{~g}$.) was added to approximately $10 \mathrm{vol}$. of sterile water, shaken vigorously, and the resulting suspension streaked upon plates of hayinfusion and dilute hay-infusion agars (Raper, 1937, 1951). Dictyostelium polycephalum was first observed several days later as diminutive fruiting structures bearing from one to five small glistening spore masses. It was hardly suggestive of a simple slime mould, but neither did it suggest any fungus with which we were familiar. Microscopic examination of the supporting stalks, composed of tiers of superimposed vacuolated cells, quickly revealed its true relationships. Isolations were made by the routine procedure of planting spores at the intersection of crossed streaks of Escherichia coli on the media cited above. The myxamoebae grew satisfactorily and the streaks of $\boldsymbol{E}$. coli were completely obliterated. The myxamoebae were observed to collect into rounded clumps from which emerged thin and variously contorted migrating pseudoplasmodia. Scattered fruiting structures were subsequently produced and these appeared, for the most part, in areas characterized by appreciable contamination with other bacteria and fungi introduced with the spore inoculum. By repeated transfer the slime mould was obtained in two-membered culture with $E$. coli but few sorocarps were produced, and these usually consisted of simple sorophores terminated by single sori. Meanwhile, parallel cultures inoculated with the mixed microflora originally associated with the slime mould continued to exhibit more complex fruiting structures in limited numbers, some of these bearing as many as 5 or 6 sori. It seemed probable that the new species might require unusual cultural conditions for optimal growth and development.

Utilizing the two-membered culture with Escherichia coli, cultivation of the slime mould was attempted upon a variety of culture media. Substrata containing relatively low concentrations $(c .0 \cdot 1 \%, \mathrm{w} / \mathrm{v})$ of glucose or lactose as a carbon source and peptone or yeast extract as a source of nitrogen supported a fair growth of the associated bacteria and permitted the slime mould to vegetate in an apparently normal if not luxuriant manner. Media containing greater concentrations of such nutrients, particularly peptone or yeast extract. were generally less satisfactory, and in no case did the slime mould develop optimally beyond the vegetative stage or an early fruiting stage characterized by the formation of abundant migrating pseudoplasmodia. Such media were not improved materially by the addition of phosphate buffers $(0.01 \mathrm{~m}$ or $0.02 \mathrm{M}$ ) as previously used for other members of the group (Raper, 1939, 1951). Fruiting structures, when present, rarely exceeded in complexity the simple 
structures previously observed with $E$. coli on the hay-infusion agars. Trials with other bacterial associates, including Aerobacter aerogenes, Serratia marcescens and Pseudomonas fluorescens, were equally unrewarding. It may be noted that in association with Serratia marcescens the sori of the slime mould remained uncoloured, hence resembling Dictyostelium mucoroides and D. minutum rather than $D$. discoideum in this regard.

By accident a plate culture of Dictyostelium polycephalum growing in association with Aerobacter aerogenes on dilute hay agar became contaminated with Dematium nigrum, ${ }^{*}$ and in the area adjacent to this yeast-like fungus numerous well-formed sorocarps developed. Attention was immediately directed toward growing the slime mould in 3-membered cultures with $\boldsymbol{A}$. aerogenes and Dematium nigrum upon a wide variety of substrata, including various modifications of thin hay agar and media containing relatively low but varying concentrations of glucose or lactose as a carbon source and peptone or yeast extract as a source of nitrogen. Of many combinations examined, maximal development of fruiting structures was obtained upon a medium that contained $0.5 \%$ glucose and $0.1 \%$ peptone solidified with $2 \%$ agar, and designated ' $0.5 \mathrm{D}-0.1 \mathrm{P}$ '. In three-membered cultures of this type incubated at $24-26^{\circ}$, growth of the slime mould was comparatively slow. Migrating pseudoplasmodia first appeared after 4-6 days and continued to form over a period of 2-3 weeks. Sorocarp formation was initiated generally after 10-14 days and continued for an additional 2-3 weeks. Pseudoplasmodial migration was often quite extensive and the coremiform fruiting structures were generally formed throughout the Petri plate with only limited concentration in zones adjacent to the sites of inoculation. A portion of such a culture is illustrated in Pl. 1 , fig. 1 , whereas pseudoplasmodia and mature fructifications formed midway between two parallel streaks seeded with the three organisms are shown, substantially enlarged, in Pl. 1, fig. 2. Equally satisfactory cultures were obtained either by streaking a mixed inoculum of the three micro-organisms on the agar plates, or by first cross-streaking the bacteria and the fungus and subsequently implanting spores or myxamoebae of the slime mould at the intersection of such streaks.

The particular advantage afforded by growing Dictyostelium polycephalum in a three-membered culture on $0.5 \mathrm{D}-0.1 \mathrm{P}$ is not immediately apparent. However, it is obvious that each of the associated micro-organisms makes its own beneficial contribution to the microcosmos wherein the slime mould thrives. In the absence of the bacteria, no apparent growth of $D$. polycephalum occurs. In the absence of the fungus, satisfactory growth of myxamoebae is readily attained and wheel-shaped aggregates commonly develop, followed by the formation of numerous migrating pseudoplasmodia. These latter structures, however, often fail to produce mature fructifications, and at most develop slight structures bearing one, two or rarely three sori. The presence of the fungus, however, in some manner renders the culture more favourable for slime mould growth; more specifically, it permits fructification of

* Identification by Dr L. J. Wickerham, Northern Utilization Research Laboratory, Peoria, Illinois. 
D. polycephalum at the optimal level thus far attained. Certain explanations suggest themselves: (1) the fungus may produce some metabolite which favours sorocarp formation; (2) it may remove or neutralize some inhibitory substance(s) produced by the growing bacteria; (3) it may alter the physical environment in which the slime mould grows; or (4) it may effect a combination of these functions, thus promoting fructification. These possibilities are now being investigated with my associate, Dr W. F. Whittingham.

Cultural conditions favouring normal fructification in any of the Acrasieae are more exacting than those required for satisfactory growth (Raper, 1956). However, in Dictyostelium polycephalum this disparity seems to be particularly accentuated. Many substrata have been employed, whereon seemingly satisfactory or even rich growth of this slime mould has been obtained followed by sparse, incomplete or aberrant fructification. Whereas such cultures leave much to be desired, they have provided a useful and, it is believed, generally accurate picture of the aggregating and migrating pseudoplasmodial stages of this species. On 0.5 D-0.1P in the presence of either Escherichia coli or Aerobacter aerogenes as the sole associate, the slime mould grows quite well at room temperature and develops aggregating pseudoplasmodia which subsequently give rise to numerous migrating bodies. However, the aggregating pseudoplasmodia in such cases are generally quite dense and of restricted dimensions, hence it is often difficult to detect the earliest stages of aggregation or to follow the inflowing streams of myxamoebae. Media containing lactose and yeast extract have proved especially useful for such observations and a formulation containing $0.5 \%$ lactose, $0.05 \%$ yeast extract and $2 \%$ agar is especially recommended ( $\mathrm{Pl}$. 2, figs. 3-5 and 9). Greater or lesser growth can be obtained by increasing the nutrient content to $1.0 \%$ lactose and $0.1 \%$ yeast extract or by decreasing it to $0.05 \%$ lactose and $0.01 \%$ yeast extract, respectively. In none of these cases, however, does optimal fruiting ensue, and fructifications, when formed, consist of small structures bearing one, two or rarely more sori. On the other hand, well-proportioned migrating pseudoplasmodia regularly develop in numbers that reflect the richness or paucity of myxamoebic growth.

The optimum temperature for Dictyostelium polycephalum appears to be about $30^{\circ}$, which is somewhat higher than that for $D$. discoideum and other species of the Dictyosteliaceae previously investigated by the writer. Excellent but less rapid growth can be obtained at lower temperatures, with migrating pseudoplasmodia first appearing after 3 weeks at $15^{\circ}$. The maximum temperature for the species appears to be in the neighbourhood of $34-35^{\circ}$.

The optimum reaction for Dictyostelium polycephalum appears to be approximately $\mathrm{pH} 6 \cdot 5$, although excellent growth of myxamoebae and the formation of abundant migrating pseudoplasmodia have been observed within the range

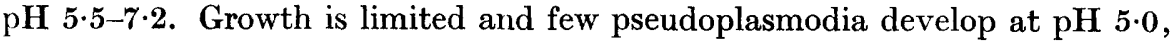
and at $\mathrm{pH} 4.5$ growth is commonly lacking. Numbers of pseudoplasmodia decrease and the pattern of those which are formed becomes increasingly atypical as the $\mathrm{pH}$ value rises above $\mathbf{7 \cdot 5}$. The $\mathrm{pH}$ value attained by any culture is strongly influenced by the composition of the substrate and the temperature 
of incubation; hence cognizance of this interdependence is essential for the successful cultivation of this slime mould, as it is for other species investigated previously. This becomes particularly important at temperatures exceeding those of the laboratory, namely $c .24-26^{\circ}$.

Additional strains of Dictyostelium polycephalum have been isolated from samples of soil collected from deciduous forests in Texas, Wisconsin, Michigan and Illinois. The species is not particularly rare, and in some collections it is quite commonplace. For example, it was isolated from seven of twenty-five samples of leaf mould and surface soil collected in September 1954 from a virginal deciduous forest at Funk's Grove, Illinois. All of these isolates have been grown in two-membered cultures in association with Escherichia coli or Aerobacter aerogenes upon most of the aforementioned media, and none of them seem to differ very markedly from the type strain, no. S-4, upon which the following account of the developmental cycle of the new species is centred.

\section{GROWTH AND MORPHOGENESIS}

\section{Spore germination}

The spores of Dictyostelium polycephalum, as in most other members of the Acrasieae, are capsule-shaped to somewhat reniform. They are quite variable in size, usually ranging from $6 \cdot 5-7 \cdot 0 \times 3 \cdot 0-3 \cdot 5 \mu$. but with some cells measuring as much as $8.0 \times 3.5 \mu$. and others as little as $4 \cdot 0-4 \cdot 5 \times 2.5 \mu$. Germination in this species is rather different from that previously observed. The spore case does not split longitudinally at one end to release an amoeboid cell. Instead, the protoplast swells and the spore wall is bulged out in a median area, becoming noticeably thinner all around this region of the spore. Eventually the wall bursts or is completely dissolved and the protoplast slowly emerges. During germination the two ends of the empty spore case may be joined at one side by a tenuous membrane, or they may become completely separated. The process is often completed within $1 \mathrm{hr}$.

\section{Vegetative growth}

Upon germination the myxamoeba of Dictyostelium polycephalum begins to feed upon the bacteria adjacent to it, enlarges and divides. As in other species, the vegetative stage consists in the independent growth and repeated division of such daughter cells until a large population of cells is built up and the available food supply is consumed, at which time the fruiting stage is initiated by the formation of aggregating pseudoplasmodia. Nothing that would distinguish these myxamoebae from those of other Dictyostelia has been observed. As seen growing on an agar surface they are very irregular in shape and size and range from broadly triangular or elongate to more or less rounded (PI. 2, fig. 7). They may or may not exhibit prominent pseudopodia. Posteriorly placed contractile vacuoles are conspicuous and a single myxamoeba may show two or three of these which normally discharge as one but may empty independently. The nucleus is difficultly discernible in unstained cells. A zone of clear ectoplasm can be seen anteriorly in cells that are rapidly moving and 
in those having extended pseudopodia. The finely granular endoplasm, comprising the bulk of the myxamoebae, shows numerous food vacuoles containing bacteria in varying stages of digestion. Whereas linear dimensions in amoeboid cells must be interpreted with caution, size ranges and proportions have some descriptive significance, and in $D$. polycephalum the vegetating myxamoebae commonly range from $5 \cdot 0-7 \cdot 5 \times 8 \cdot 0-12 \cdot 0 \mu$. with seemingly larger and smaller cells not uncommon.

Under optimal conditions for growth and development, the vast majority of the vegetative myxamoebae aggregate to form pseudoplasmodia at the close of the vegetative stage and eventually these, or their daughter cells, differentiate to form the structural elements, stalk cells and spores, of mature sorocarps. However, in most cultures limited numbers of more or less isolated myxamoebae fail to enter the wheel-like aggregates and instead round up as individual cells, become encased by somewhat rigid cellulose walls to form microcysts, and in this condition enter a temporary to prolonged resting stage. In certain cultures which are suboptimal for one reason or another, the greater portion of the free-living vegetative population forms microcysts and never enters the normal fruiting process. This phenomenon is often particularly marked in cultures grown with Aerobacter aerogenes on substrates containing lactose and yeast extract, and seemingly it is most pronounced in the more central areas of broad bands of mixed bacteria and slime mould growth. Individually the microcysts are spherical or nearly so, measure approximately $4 \cdot 0-6 \cdot 5 \mu$. and germinate by a simple process of excystment whereby the wall of the microcyst splits open and the amoeboid protoplast emerges to renew its vegetative cycle.

\section{Pseudoplasmodium formation}

The phenomenon of myxamoebic aggregation, or pseudoplasmodium formation, in Dictyostelium polycephalum is basically similar to that in species already known. With the exhaustion of the available food supply (bacteria) the myxamoebae elongate, become uniformly oriented and stream toward centres of aggregation presumably in response to some chemotactic stimulus, as in D. discoideum (Bonner, 1947; 1949), which is first produced by the centrally placed myxamoebae and subsequently by those comprising the radiating arms of inflowing cells. During this process the myxamoebae crowd together and assume a characteristic limax form, their dimensions commonly ranging from 6-4 $\times 15-20 \mu$. (Pl. 2, fig. 8). Such aggregating cells are characterized by conspicuous anterior zones of non-granular ectoplasm, inconspicuous food vacuoles, and contractile vacuoles which continue their rhythmic enlargement and discharge. In contrast to D. discoideum, D. mucoroides and D. purpureum, where conspicuous radiating streams usually develop soon after the centres of aggregation become apparent, the developing pseudoplasmodia of $D$. polycephalum may for several hours remain evident only as areas of somewhat greater cell concentration surrounding small but definitely raised centres. Superficially, the extent of the nascent aggregate may be indicated by a more or less well-defined halo as seen in Pl. 2, fig. 3. During this stage, and for 
some time thereafter, the myxamoebae normally continue to aggregate as broad and nearly continuous, sheets of inflowing cells that more or less completely surround the point of aggregation. As aggregation progresses, prominent and characteristic wheel-like patterns usually emerge, with the radiating streams often tending to terminate abruptly, as seen in Pl. 2, fig. 4. In other cases, discrete streams are more sharply defined and are strongly anastomosed (Pl. 2, fig. 5), whereas in still others the pseudoplasmodia may persist for a longer time as broad inflowing streams which at times exhibit concentric, or transverse, bands of alternately crowded and thinly disposed cells, probably indicating a wave-like progression of the aggregating cells (Pl. 2, fig. 6). The developing pseudoplasmodia commonly attain a diameter of $5-7 \mathrm{~mm}$. As aggregation proceeds, the central area becomes papillate and from each of the papillae arises a long thin upright column of myxamoebae. These columns continue to develop simultaneously with the influx of myxamoebae from the peripheral areas of the aggregates, and in time become several millimetres in length, topple over so that they rest on the surface of the agar and begin a slow migration outward from the centres where they were formed (Pl. 2, fig. 9).

\section{Migrating pseudoplasmodia}

The migrating pseudoplasmodia of Dictyostelium polycephalum must, I think, be considered comparable to those produced in $D$. discoideum (Raper, 1935, et seq.); nevertheless, they differ from the latter in a number of significant particulars. First, as many as a dozen or more migrating bodies may arise from a single aggregation centre. Secondly, they are, by comparison, quite long and very thin, exhibit no apparent response to light or temperature, and show a markedly greater tendency to become transversely segmented. Thirdly, no migrating pseudoplasmodium has ever been observed to divide dichotomously nor have two of these structures been seen to coalesce, thereby forming either a smaller or larger, but otherwise normal, fruiting organization as in $\boldsymbol{D}$. discoideum. Finally, each migrating body is potentially capable of producing a number of sorocarps which develop simultaneously and together constitute a coremiform fructification, whereas in $D$. discoideum a single migrating body, of whatever origin, typically gives rise to a single sorocarp (Raper, $1940 a$; $1941 a$ ).

The migrating pseudoplasmodia of Dictyostelium polycephalum merit further consideration. These bodies commonly attain a length of 5-10 mm. or more, while they seldom exceed $50-60 \mu$. in diameter. They range from almost straight to irregularly curved and twisted in form (Pl. 3, figs. 10, 11), depending in part at least upon the relative rates of progression in different areas of the migrating masses. 'They may be essentially uniform in diameter throughout, or they may show relatively thick and thin areas, and in extreme cases may present a strikingly beaded appearance, the significance of which is not at present known. Migration presents some interesting problems. It is not uncommon to observe a migrating pseudoplasmodium which is in contact with the agar only at its posterior end, or to see an elongate body which touches the substrate at only two or three points throughout its entire length, or again to see one moving 
through the aerial hyphae of some accompanying fungus as illustrated in Pl. 3, fig. 12, where a species of Absidia has been introduced into the culture vessel. When moving on agar the elongate pseudoplasmodium commonly becomes thickened at points of contact with the substrate, the enveloping slime sheath becomes ruptured, and the migrating body becomes severed at these sites. The intact portion anterior to such a break will normally continue its migration uninterrupted. The posterior portion may develop a new apical tip and resume migration, or the disintegrative process may continue further with the myxamoebae separating from each other. In the presence of bacteria these will return to the vegetative stage, and in their absence they may or may not reorganize into new and smaller fruiting organizations. The internal organization of the migrating pseudoplasmodium is obviously less highly developed than in Dictyostelium discoideum. Whereas careful histological studies still remain to be made, there is no evidence to indicate areas of presumptive specialization into pre-stalk and pre-spore myxamoebae, a condition that is not surprising when one recalls that each of these structures is potentially capable of subsequently subdividing to produce several sorogens. Viewed microscopically, only the myxamoebae that comprise a minute anterior fraction $(c .50 \pm \mu$.$) exhibit a transverse orientation.$

Under optimal conditions the migrating pseudoplasmodia may travel for several centimetres before halting to form sorocarps. The rate of migration is generally about $0.5 \mathrm{~mm}$. $/ \mathrm{hr}$. in contrast to $1.0-2.0 \mathrm{~mm}$. $/ \mathrm{hr}$. for Dictyostelium discoideum (Raper, 1940a).

The migrating pseudoplasmodia of Dictyostelium polycephalum appear to exhibit little or no response to light. This is in marked contrast to $D$. discoideum where light is a principal stimulus governing pseudoplasmodial migration, and to $D$. mucoroides, D. purpureum and Polyspondylium violaceum where light so strikingly determines the length of the sorophores and the direction in which sorocarps are built (Raper, 1940a, $b, 1941 a$ ).

\section{Sorocarp formation}

Following varying periods and distances of migration the pseudoplasmodia cease forward movement and collect into compact masses preparatory to sorocarp formation. Typically, such a mass gradually invaginates vertically into a number of contiguous but more or less well-defined columns of myxamoebae, each of which will normally build an individual sorocarp. The details of this process have not yet been investigated carefully; nevertheless, sufficient observations have been made to follow the main course of events. Stalk formation is initiated within each of the columns prior to any obvious elongation of these, and as this proceeds the individual sorogens become apparent as they are slowly raised above the substratum (Pl. 4, fig. 13). With the continued elongation of the individual sorophores, the sorogens, or columns of undifferentiated myxamoebae, become increasingly distinct and tend to diverge from one another as seen in Pl. 4, fig. 14. During this period an individual sorophore is being formed within the terminal area of each of these, and yet the processes of cellular differentiation within the several sorogens is sufficiently inter- 
dependent so that all of the sorophores are constructed at a nearly uniform rate. It is at present a noteworthy and perplexing fact that whereas the sorophores are lengthening at divergent sites, the completed stalks are in the majority of cases closely appressed and to some degree cemented together to form a wellproportioned, multi-stalked support. This picture changes abruptly when the sorophores reach $75-80 \%$ of their ultimate length, for beyond this stage they cease to be drawn together and each retains its original orientation until sorophore formation is completed. The capsule-shaped spores are produced coincident with this latter stage of stalk formation, and each sorophore terminates in a globose sorus of dimensions proportional to the mass of myxamoebae contributing to its formation. The net result of the morphogenetic changes just outlined is the construction of compound fruiting structures, which consist of a variable number of distinct but conjoined sorocarps. The general habit of such fructifications is shown in Pl. 1, fig. 2, and in Pl. 4, fig. 16; details of their cellular structure can be seen in Pl. 5, fig. 20. Such coremiform fruiting structures may contain as many as eight or ten individual sorocarps, although structures containing from two to six are much more commonplace, and in every culture, however favourable, numerous sorocarps bearing single sori are regularly formed. Except for the absence of a basal disk, such simple sorocarps bear a striking resemblance to the diminutive but otherwise normal fruiting structures found in very thin cultures of Dictyostelium discoideum. When reduced to its simplest terms, the formation of compound fruiting structures in $D$. polycephalum may thus be interpreted as the co-ordinated and contiguous development of two or more small unbranched sorocarps.

Microscopic examination readily reveals that the polycephalic fruiting structures of Dictyostelium polycephalum represent clusters of discrete sorocarps, and this is clearly shown in Pl. 5. It will be noted that the individual sorophores consist, for the most part, of single tiers of superimposed and strongly vacuolated cells, a structural pattern which is duplicated by other species of Dictyostelium in the construction of very small sorocarps. What distinguishes D. polycephalum particularly is this: when more myxamoebae are present in a migrating pseudoplasmodium than can be used for the construction of a single sorocarp of the limited dimensions that characterize the species, that fruiting organization responds not by building a larger sorocarp but by dividing into a variable number of parts, each of which builds its own sorocarp of these same general dimensions. Significantly, this concurrent building of multiple sorocarps is far from haphazard. The processes of pseudoplasmodial invagination and subsequent cellular differentiation obviously reflect a considerable measure of community control, otherwise the component parts would not develop at uniform rates, the several stalks would not adhere together, and the constituent sorocarps would be expected to show a much greater diversity in form and size. That a very considerable degree of uniformity exists among the sorocarps that comprise a particular coremiform structure, and to a lesser degree between these and the sorocarps of other compound fructifications, is indicated in Table 1. In this representative tabulation 
certain selected measurements were recorded for a number of fructifications of varying complexity observed in a small area of a favourable culture of the type illustrated in Pl. 1. Two simple sorocarps with single sori are included for comparison. The overall height of these fructifications varied only from $\mathbf{3 5 0}$ to $650 \mu$. with extremes of 40 and $90 \mu$. being occasionally observed in the diameters of their sori. It is regarded as particularly significant that the length

\section{Table 1. Proportions of the coremiform fructifications of Dictyostelium polycephalum}

Selected measurements of representative fructifications composed of different numbers of sorocarps produced in a limited are $i$ of a three-membered culture of Dictyostelium polycephalum grown in association with Lerobacter aerogenes and Dematium nigrum on an ar medium containing $0.5 \%+0 \cdot 1 \%$ peptone. Incubation was at $c .24-26^{\circ}$ for 3 weeks.

\begin{tabular}{|c|c|c|c|c|c|c|}
\hline \multirow{3}{*}{$\begin{array}{l}\text { Overall } \\
\text { height of } \\
\text { fructifi- } \\
\text { cation } \\
(\mu .)\end{array}$} & \multirow{2}{*}{\multicolumn{2}{|c|}{ Sori }} & \multicolumn{4}{|c|}{ Dimensions of compound stalk } \\
\hline & & & \multirow[b]{2}{*}{$\begin{array}{c}\text { Dia- } \\
\text { meter } \\
\text { at base } \\
(\mu .)\end{array}$} & \multirow{2}{*}{$\begin{array}{l}\text { Dia- } \\
\text { meter } \\
\text { at mid- } \\
\text { point } \\
(\mu .)\end{array}$} & \multirow{2}{*}{$\begin{array}{l}\text { Length: } \\
\text { base to } \\
\text { diver- } \\
\text { gence } \\
(\mu .)\end{array}$} & \multirow[b]{2}{*}{$\begin{array}{c}\text { Length: } \\
\text { divergence } \\
\text { to termini } \\
(\mu .)\end{array}$} \\
\hline & $\begin{array}{l}\text { No. per } \\
\text { fructifi- } \\
\text { cation }\end{array}$ & $\begin{array}{l}\text { Diameters of } \\
\text { individual sori } \\
\left(\mu_{.}\right)\end{array}$ & & & & \\
\hline 400 & 1 & 75 & 20 & 10 & - & None \\
\hline 450 & 1 & 90 & 20 & 12 & - & None \\
\hline 475 & 2 & 45,70 & 18 & 12 & 375 & $75-125$ \\
\hline 350 & 2 & 60,65 & 20 & 15 & 275 & $75-100$ \\
\hline 550 & 3 & $50,60,60$ & 40 & 20 & 425 & $100-125$ \\
\hline 500 & 3 & $65,60,65$ & 25 & 12 & 400 & $75-100$ \\
\hline 475 & 3 & $50,45,50$ & 25 & 15 & 375 & $90-100$ \\
\hline 375 & 3 & $40,45,45$ & 15 & 12 & 275 & $90-100$ \\
\hline 500 & 3 & $80,70,65$ & 30 & 15 & 350 & $125-150$ \\
\hline 500 & 4 & $45,50,50,55$ & 35 & 15 & 400 & $90-100$ \\
\hline 625 & 4 & $90,80,80,75$ & 30 & 20 & 475 & $125-150$ \\
\hline 550 & 4 & $70,75,70,85$ & 35 & 20 & 400 & $110-150$ \\
\hline 650 & 5 & $60,75,60,70$ & 40 & 25 & 500 & $125-150$ \\
\hline 550 & 5 & $40,40,50,60,45$ & 45 & 25 & 450 & $90-100$ \\
\hline 575 & 5 & $80,70,80,85,65$ & 45 & 30 & 425 & $125-150$ \\
\hline 525 & 6 & $60,60,55,60,45,50$ & 30 & 15 & 400 & $100-125$ \\
\hline 600 & 7 & $\begin{array}{l}70,60,70,65,75,55 \\
60\end{array}$ & 50 & 30 & 475 & $100-150$ \\
\hline 650 & 7 & $50,50,55,60,55,60$ & 60 & 30 & 500 & $125-150$ \\
\hline 550 & 9 & $\begin{array}{l}50,45,40,45,45,50 \\
60,40,45\end{array}$ & 55 & 30 & 400 & $125-150$ \\
\hline
\end{tabular}

of the sorophores from the points of divergence to their apices generally represented approximately $\mathbf{2 0 - 2 5} \pm \%$ of their total length. It can hardly be doubted that this consistent proportion reflects a considerable degree of heritable specificity in the control of the morphogenetic processes.

Considered individually, the sorophores show an overall and generally consistent taper from their bases to a site just below the level of divergence, and this is reflected in the corresponding diameter of the compound stem. At their bases the larger sorophores may show two or even three cells in cross-section whereas at their mid-points they usually consist of single rows of cells, and in more terminal areas this is almost: invariably the case. Occasionally, one or 
more of the sorophores that comprise a coremiform support may be initiated as completely independent entities (Pl. 5, fig. 19). However, as sorophore formation progresses these become increasingly appressed to form a quite rigid unitary support. In the subterminal area of divergence the sorophores thicken substantially, and at this level the diameter of one of these may approach that of the whole bundle at a somewhat lower level. These differences result from the varied proportions assumed by constituent vacuolated cells at different stages in the morphogenesis of the compound fructification, and they can be assumed to reflect a response to the greater loads which must be supported by the individual sorophores as they develop outward from a previously common vertical axis.

\section{DISCUSSION}

Dictyostelium polycephalum presents many interesting and unique features in its fruiting phase. The sorocarps are quite diminutive, rarely exceeding $650 \mu$. in length, and considered individually they are somewhat smaller than those normally formed by any other known member of the genus. Viewed as coremiform clusters, in which form they characteristically occur, they appear to be branched at a single point some distance below the level of the sori. This is in marked contrast to all other species of Dictyostelium where branching is either lacking or occurs in an irregular and haphazard fashion, and upon this basis alone one might question the wisdom of placing the new slime mould in this genus. However, when viewed microscopically, the apparent unitary fructification is seen to consist of a number of discrete sorocarps, i.e. individual fruiting structures, the greater portion of whose sorophores are closely adherent but whose sorus-bearing apices are strongly divergent. Comparable fructifications are not found in any other described member of the Acrasieae, although structures somewhat suggestive of them were apparently observed in two genera, Acrasis and Coenonia, described by van Tieghem in 1880 and 1884, respectively. Whereas he published no illustrations and neither genus has been since reported, his observations on the life cycles of the Acrasieae were most penetrating and his descriptive accounts are sufficiently clear to leave little doubt concerning the overall aspect of the slime moulds that he studied. Acrasis was described as sometimes showing a coremiform habit, but the constituent stalks bore chains of spherical and slightly roughened spores rather than sori containing smooth-walled spores of the type found in other forms. The second genus, Coenonia, was reported as producing branched fruiting structures when grown in rich culture and, if my interpretation is correct, the branches generally arose at a single level on the upright sorophore. Such a structure, viewed as a whole, might conceivably resemble the compound fructifications of Dictyostelium polycephalum, although there is nothing in van Tieghem's account to indicate a coremiform support. The overall dimensions of van Tieghem's Coenonia were considerably greater than those reported for the new slime mould, and each branch was reported to terminate in a cupule which supported and partially enclosed a gelatinous mass of spores. Obviously, Dictyostelium polycephalum cannot be regarded as closely related to either of 
these long-missing slime moulds, but it is believed significant that in its fructifying processes it does exhibit morphogenetic manifestations which are in some measure remindful of van Tieghem's genera. Branched fructifications constitute, of course, the chief distinguishing characteristic of the ubiquitous genus Polysphondylium (Brefeld, 1884; Harper, 1929). In this case the fructification unquestionably represents a single unitary sorocarp, just as is believed to be the case in Coenonia. In Polysphondylium the rising sorogen repeatedly casts off and leaves behind on the lengthening sorophore small masses of myxamoebae which subsequently segment, giving rise to whorls of lateral branches that are anchored to the sorophore and attain maturity coincident with the continued construction and elongation of the main axis. In Coenonia the branching habit is suspected of resulting from the segmentation of a single mass of undifferentiated myxamoebae midway during the process of sorocarp construction. The characteristic branching of Polysphondylium, and presumably of Coenonia as well, is then in no true sense comparable with the pseudo-branching which is such a distinguishing feature of Dictyostelium polycephalum, for in the latter case vertical segmentation of the pseudoplasmodial body occurs soon after it ceases migration and precedes any beginnings of sorocarp formation.

Certain other species of Dictyostelium regularly produce a variable number of sorocarps from a single aggregating pseudoplasmodium. This is particularly true of the other diminutive species, D. minutum and D. lacteum, and to a lesser degree in the larger members of this genus and in Polysphondylium as well. No other species is known, however, where the sorophores of two or more individual sorocarps are normally adherent. In Dictyostelium minutum it is not uncommon to observe a half-dozen or more sorocarps to arise from a single aggregate, and in $D$. lacteum this number is often exceeded. The formation of such sorocarps is regularly initiated while myxamoebic aggregation is still in progress, and each sorocarp obviously develops independently of all the others despite their common origin from a single fractionating aggregate. The gregarious sorocarps arising from a primary pseudoplasmodium in these species thus represent a group, or cluster, of fructifications in which the morphogenetic changes leading to culmination are completely independent in the different sorocarps. The situation in D. polycephalum is quite different from this: (1) a migrating stage, which is lacking from these other species, is regularly interposed between the stages of aggregation and sorocarp formation; (2) upon the cessation of migration the pseudoplasmodial mass segments more or less equally along vertical planes prior to the initiation of sorophore formation; (3) these nascent sorogens are basically separate from one another but are contiguous and remain interdependent so that culmination tends to proceed uniformly in all of them; (4) the sorophores which they form are constructed so that they constitute a single, rigid supporting column until they have attained approximately three-quarters of their ultimate height. Only in Acrasis, and possibly in Coenonia, among the reported cellular slime moulds does an even remotely similar pattern of morphogenesis occur, and for these genera the surmised parallelism in behaviour is highly speculative, for 
the completed fructifications in both genera differ markedly in other particulars from those of Dictyostelium polycephalum.

A migrating pseudoplasmodial stage regularly occurs in only one other species of the Acrasieae, Dictyostelium discoideum, and between this and the migrating pseudoplasmodium of $\boldsymbol{D}$. polycephalum marked differences occur in form, in structure, and in behaviour. Whereas more than one migrating body can and often does arise from a single aggregating pseudoplasmodium in D. discoideum, the more usual behaviour is for such a wheel-like aggregate to produce a single, motile, slug-like body and for this in turn to build a single sorocarp. An individual slug may split longitudinally to produce two smaller but otherwise typical migrating bodies, or two migrating structures of separate origins may collide and fuse to form a larger and equally typical migrating pseudoplasmodium, and in either case the migrating pseudoplasmodium regularly forms a single sorocarp of characteristic form and of dimensions proportional to the mass of myxamoebae present in the organization at the time of culmination (Raper, 1940a). The migrating pseudoplasmodia of $\boldsymbol{D}$. discoideum show a strong axial polarity and exhibit striking and highly sensitive responses to light and temperature. In contrast, the aggregating pseudoplasmodium of $\boldsymbol{D}$. polycephalum regularly gives rise to many migrating bodies, each of which is potentially capable of producing a coremiform fructification consisting of several sorocarps. In so far as we have observed, the migrating bodies of this species neither split nor fuse to form larger or smaller migrating units.

The form and structure of the migrating structures in the two species are quite different. Whereas the proportions of length to width of the slugs in Dictyostelium discoideum vary in response to cultural conditions and other factors, one seldom observes actively moving bodies where this proportion exceeds $10: 1$, while it is commonplace to see migrating bodies of $D$. polycephalum where the same proportions are $100: 1$, or even greater. By comparison, the migrating bodies of the latter species are very long and thin. As in D. discoideum they are bounded by an envelope of slime, termed the slime sheath, that is secreted by the mass of myxamoebae, and during migration this is deposited on the agar surface as a continuous hyaline slime track. The envelope is obviously very tenuous and the track is extremely delicate. Possibly the insubstantial nature of the slime sheath of $D$. polycephalum accounts in part for the very elongate form typically assumed by the migrating bodies; certainly it could account for the marked tendency of the slugs of this species to break up into transverse segments, and in some cases to disintegrate as organizational entities, thereby releasing their constituent myxamoebae. The migrating pseudoplasmodium of $D$. polycephalum, like that of $D$. discoideum, shows a definite anterior end and the movement of the whole mass is apparently guided by it, although such movements appear to be at random rather than highly directed as in $D$. discoideum. Structurally, the pseudoplasmodium of D. polycephalum is composed of relatively few cells in transverse section because of its limited diameter, and throughout the greater portion of the body the constituent myxamoebae seem to have little distinctive form or 
orientation. There is evidence that some of the peripheral cells are elongated in the direction of movement, and it is thought that the areas of the pseudoplasmodium where these occur are at the moment probably progressing more rapidly than other areas or, for that matter, the body as a whole. The myxamoebae in the extreme anterior region are characteristically oriented in the direction opposite to that of pseudoplasmodial movement. This, it is believed, is comparable to the situation in $D$. discoideum, but the area of transverse cellular orientation is much less extensive (see Bonner, 1944). Such directed action as the migrating body of $D$. polycephalum displays is believed to stem from this area.

It is in the process of sorocarp formation, or culmination, that Dictyostelium polycephalum exhibits its greatest difference from other members of the genus. It is noteworthy that the migrating pseudoplasmodium characteristically subdivides into a number of sorogens when it comes to rest preparatory to fructification; it is remarkable that these sorogens once formed remain sufficiently interdependent to culminate at a uniform rate; it is most extraordinary that the sorophores which are constructed individually by these sorogens should be formed contiguous to one another and that they should be cemented together to form a compound and, therefore, more rigid support for the elevation of the spore-bearing sori.

Lyophilized cultures of Dictyostelium polycephalum growing in association with Dematium nigrum and Aerobacter aerogenes have been deposited in the American Type Culture Collection, Washington, D.C., and in the Centraalbureau voor Schimmelcultures, Baarn, Holland.

This investigation was supported in part by a research grant, NSF-C 612, from the National Science Foundation and in part by a research grant, C-2119 (C) M and G, from the National Cancer Institute, of the National Institutes of Health, Public Health Service.

The writer is indebted to Miss Dorothy Fennell, former associate at the Northern Regional Research Laboratory (Peoria, Illinois), for her aid in the isolation and early cultural studies of this slime mould, to Miss Joan Cormier and Mrs Bessie LaBudde for assisting in the preparation of the illustrations, to Claude $\mathbf{E}$. Vezina for preparing the Latin diagnosis, and to Miss Twila Strocher for performing preliminary studies on pH and temperature relationships.

\section{REFERENCES}

Bonner, J. T. (1944). A descriptive study of the development of the slime mold Dictyostelium discoideum. Amer. J. Bot. 31, 175.

Bonner, J. T. (1947). Evidence for the formation of cell aggregates by chemotaxis in the development of the slime mold Dictyostelium discoideum. J. exp. Zool. 106, 1.

Bonner, J. 'T. (1949). The demonstration of acrasin in the later stages of the slime mold Dictyostelium discoideum. J. exp. Zool. 110, 259.

Brefeld, O. (1869). Dictyostelium mucoroides. Ein neuer Organisms aus den Myxomyceten. Abh. senckenb. naturf. Ges. 7, 85.

Brefeld, O. (1884). Polysphondylium violaceum und Dictyostelium mucoroides nebst Bemerkungen zur Systematik der Schleimpilze. Untersuchungen aus dem Gesammtgebiete der Mykologie, Pt. 6, 1. 
Harper, R. A. (1929). Morphogenesis in Polysphondylium. Bull. Torrey bot. Cl. 56, 227.

Olive, E. W. (1901). A preliminary enumeration of the sorophoreae. Proc. Amer. Acad. Arts Sci. 37, 333.

Olıve, E. W. (1902). Monograph of the Acrasieae. Proc. Boston Soc. nat. Hist. 30, 451.

RAPER, K. B. (1935). Dictyostelium discoideum, a new species of slime mould from decaying forest leaves. J. agric. Res. 50, 135.

RAPER, K. B. (1937). Growth and development of Dictyostelium discoideum with different bacterial associates. J. agric. Res. 55, 289.

RAPER, K. B. (1939). Influence of culture conditions upon the growth and development of Dictyostelium discoideum. J. agric. Res. 58, 157.

RAPER, K. B. (1940a). Pseudoplasmodium formation and organization in Dictyostelium discoideum. J. Elisha Mitchell sci. Soc. 56, 241.

RAPER, K. B. $(1940 b)$. The communal nature of the fruiting process in the Acrasieae. Amer. J. Bot. 27, 436.

RAPER, K. B. (1941 a). Developmental patterns in simple slime moulds. Growth, 5, 41 .

RAPER, K. B. (1941 b). Dictyostelium minutum, a second new species of slime mould from decaying forest leaves. Mycologia, 33, 633.

RAPER, K. B. (1951). Isolation, cultivation, and conservation of simple slime moulds. Quart. Rev. Biol. 26, 169.

RAPER, K. B. (1956). Factors affecting growth and differentiation in simple slime moulds. Mycologia.

Singh, B. N. (1947). Studies on Soil Acrasieae. I. Distribution of species of Dictyostelium in soils of Great Britain and the effect of bacteria on their development. J. gen. Microbiol. $1,11$.

Tieghem, Ph. van (1880). Sur quelques myxomycètes à plasmode agrégé. Bull. Soc. bot. Fr. 27, 317.

Tieghem, Ph. van (1884). Coenonia, genre nouveau de myxomycètes à plasmode agrégé. Bull. Soc. bot. Fr. 31, 303.

\section{EXPLANATION OF PLATES}

$$
\text { Plate } 1
$$

Fig. 1. Dictyostelium polycephalum growing on glucose $(0.5 \%)+$ peptone $(0 \cdot 1 \%)$ agar medium in association with Aerobacter aerogenes and Dematium nigrum; incubation 3 weeks at $24-26^{\circ} . \times 2$.

Fig. 2. Sorocarps and newly formed migrating pseudoplasmodia on an agar block (from fig. 1) viewed from the side. $\times 40$.

\section{Plate 2}

Pseudoplasmodium formation in Dictyostelium polycephalum growing in two-membered culture with Escherichia coli on agar containing lactose (0.5\%) + yeast extract (0.05\%).

Fig. 3. Early aggregating stage: centre of pseudoplasmodium appears as a dark ring, whereas the extent of aggregation is indicated by the broad halo surrounding this. $\times 10$.

Fig. 4. A more advanced stage showing well-formed inflowing streams of myxamoebae. $\times 10$.

Fig. 5. Fully developed aggregating pseudoplasmodium with anastomosing streams of myxamoebae. $\times 10$.

Fig. 6. Aggregating pseudoplasmodium developing at colony margin; concentric bands are believed to result from wave-like convergence of myxamoebae. $\times 20$.

Fig. 7. Unoriented, free-living vegetative myxamoebae. $\times 300$.

Fig. 8. Portion of a pseudoplasmodial stream (as in fig. 5) showing uniformly oriented myxamoebae flowing downward toward the aggregation centre which is outside the picture. $\times 300$.

Fig. 9. Long, thin migrating pseudoplasmodia emerging from two centres of aggregation. $\times 10$. 
Plate 3

Migrating pseudoplasmodia of Dictyostelium polycephalum

Fig. 10. Migrating pseudoplasmodia moving away from the sites where they originated. $\times 15$.

Fig. 11. Two well-formed migrating pseudoplasmodia. $\times 25$.

Fig. 12. A migrating pseudoplasmodium moving through the aerial mycelium of Absidia coerulia; note that this is supported at only three points throughout its entire length. $\times 15$.

\section{Plate 4}

Sorocarp formation in Dictyostelium polycephalum

Fig. 13. Early stage in the formation of a coremiform fructification. The migrating pseudoplasmodium has become subdivided into several, vertically oriented sorogens, each of which will build a sorocarp side view. $\times 75$.

Fig. 14. A more advanced stage in a different fructification. $\times 60$.

Fig. 15. The coremiform fructification, consisting of 7 sorocarps, developed from the cluster of sorogens shown in fig. 13, photographed one day later. $\times 75$.

Fig. 16. Mature fructifications showing their general form and habit. $\times 40$.

\section{Plate 5}

Coremiform fructifications of Dictycstelium polycephalum stained with rose bengal in $5 \%$ aqueous phenol and photographed in situ

Fig. 17. A single sorocarp with its single globose sorus. $\times 50$.

Fig. 18. Coremiform fruiting structure composed of 3 sorocarps. $\times 50$.

Fig. 19. Compound structure composed of 5 sorocarps. $\times 150$.

Fig. 20. Coremiform fructification showing cellular detail in its three adherent sorophores. The basal apron of slime anchor: the structure to the substratum and holds it in an upright position. $\times \mathbf{2 7 5}$. 
Journal of General Microbiology, Vol. 14, No. 3
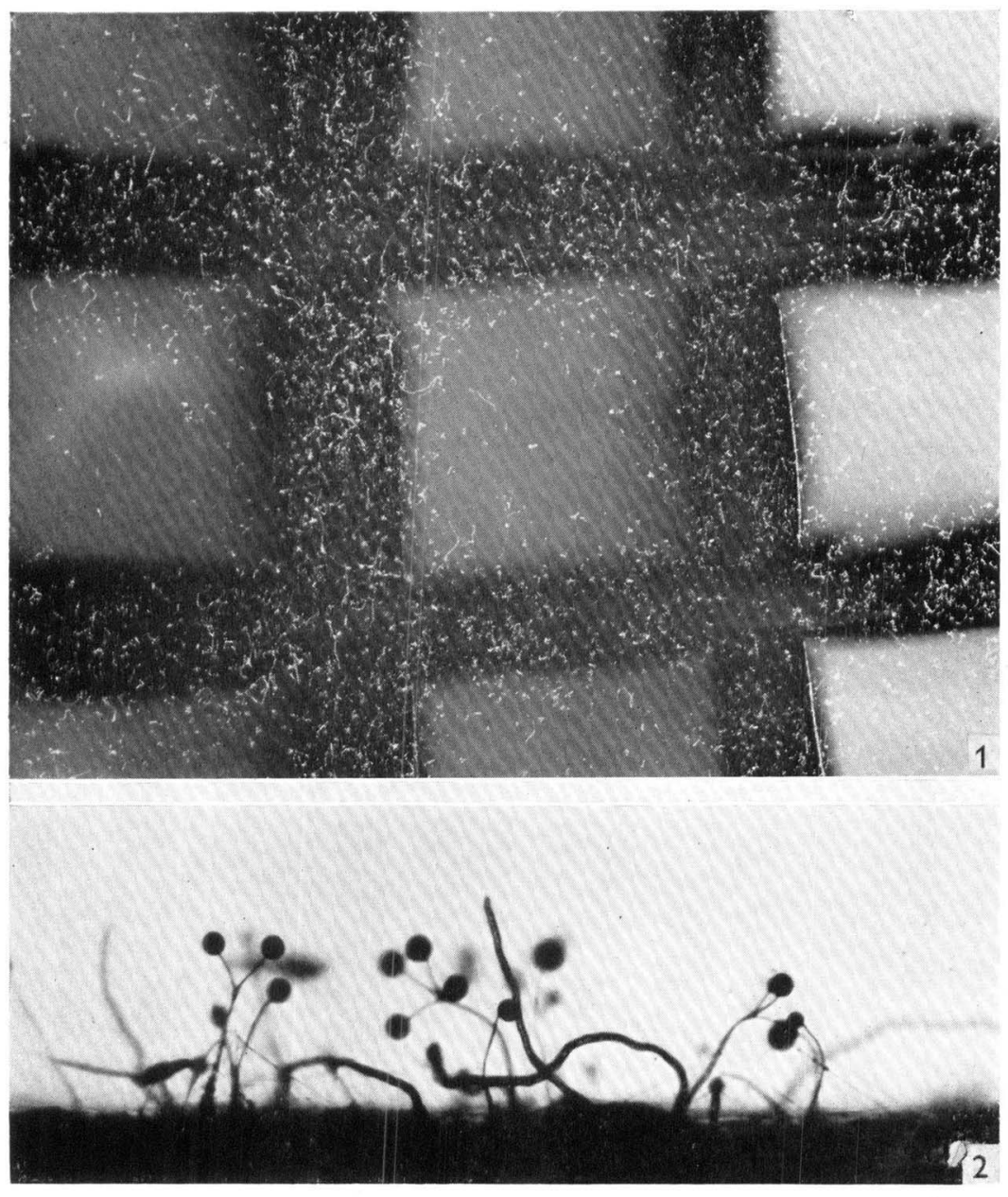

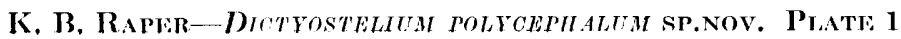

(Facing $p .732)$ 
Journal of General Microbiology, Vol. 14, No. 3
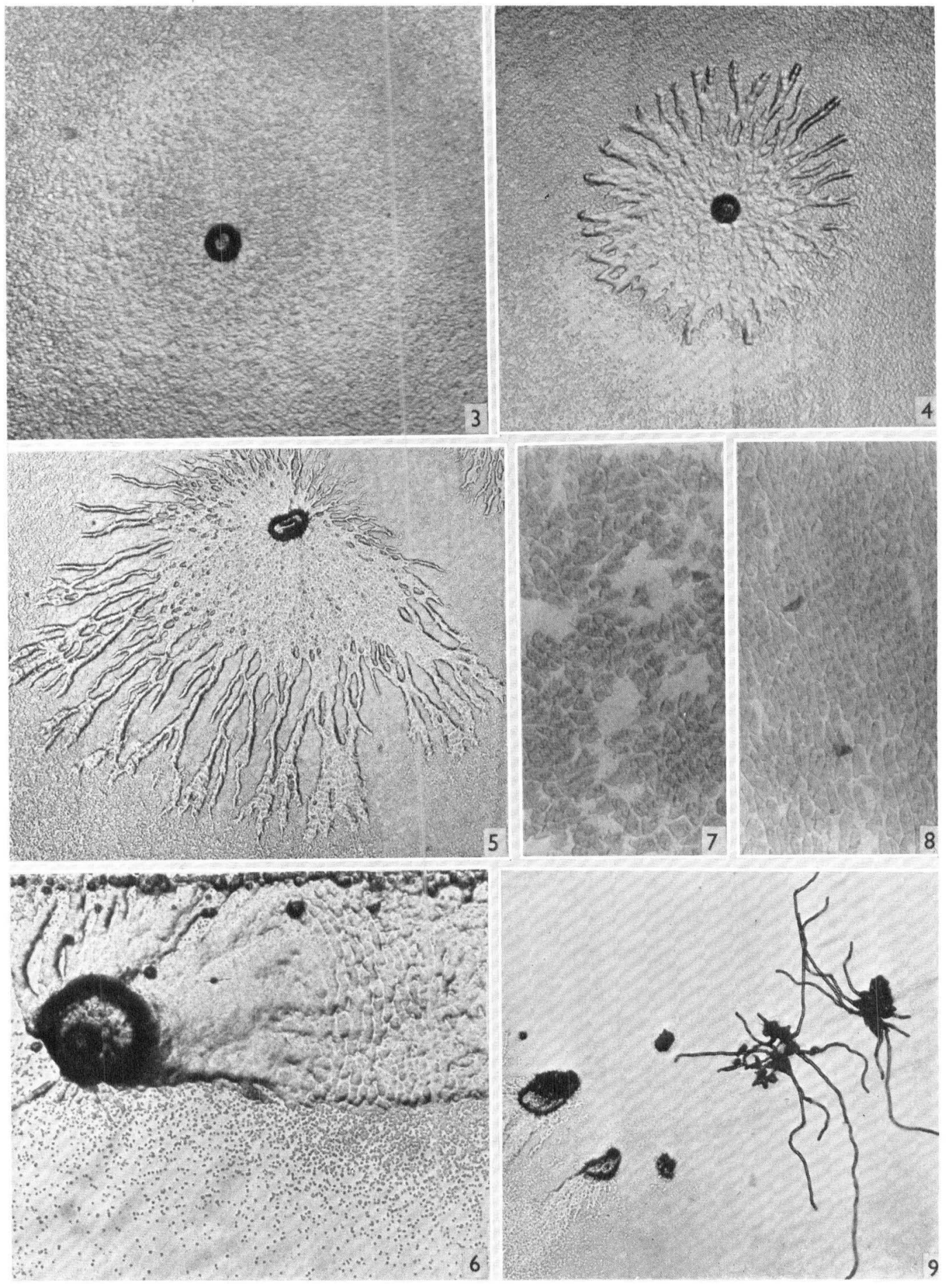

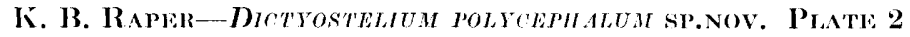


Journal of General Microbiology, Vol. 14, No. 3
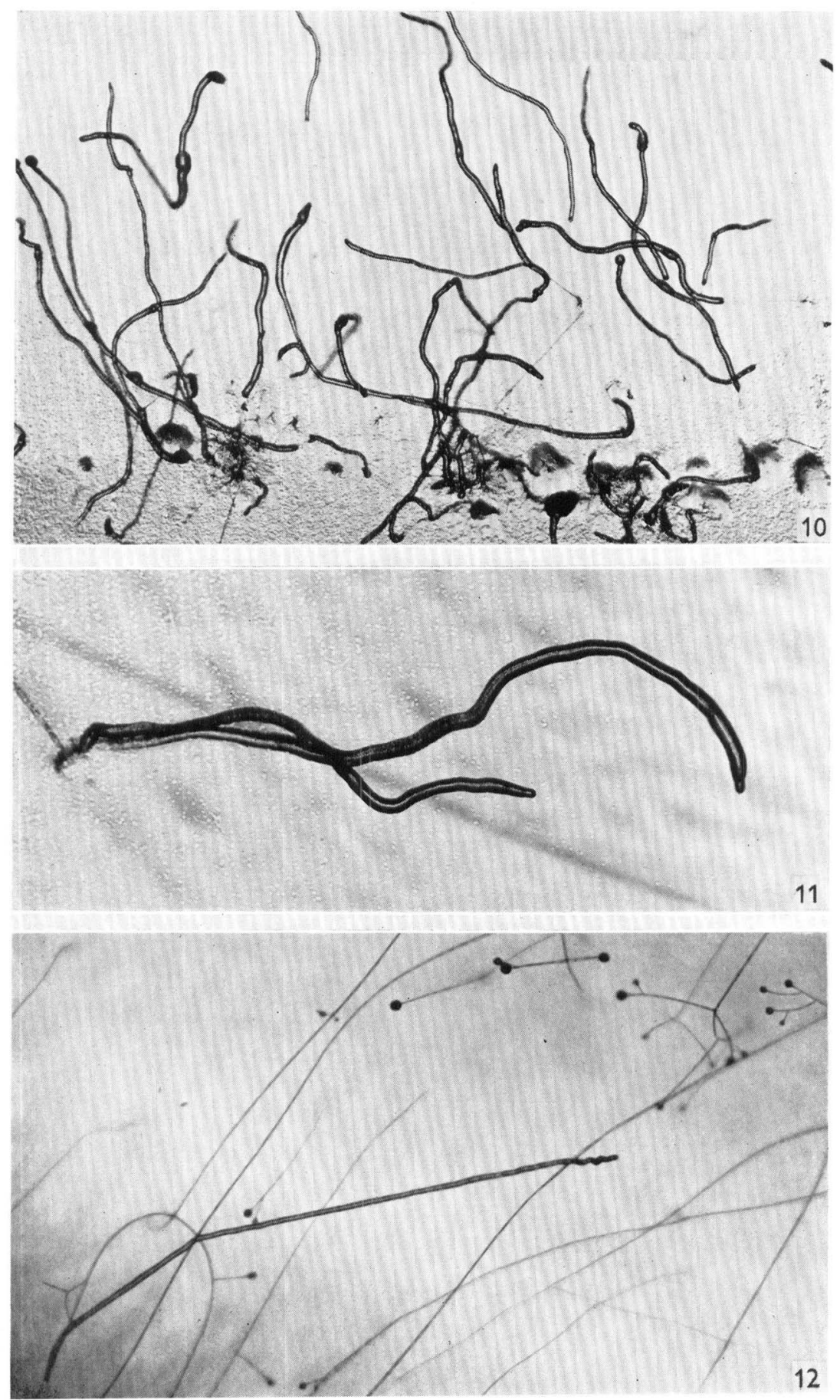

K. B. Raper-Dictyostellum polychphalum sp.nov. Plate 3 
Journal of General Microbiology, Vol. 14, No. 3
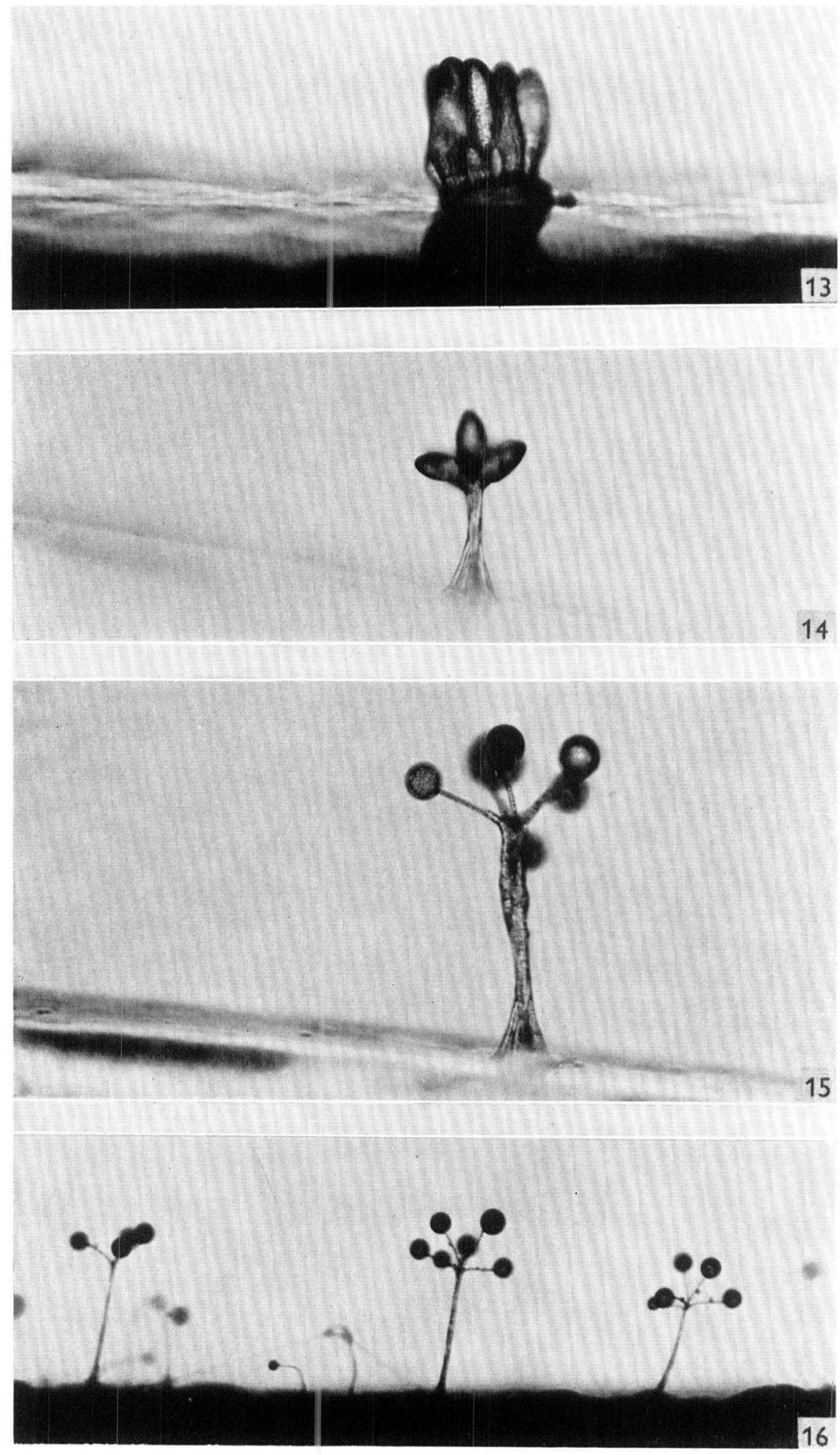

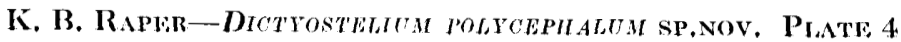


Journal of General Microbiology, Vol. 14, No. 3
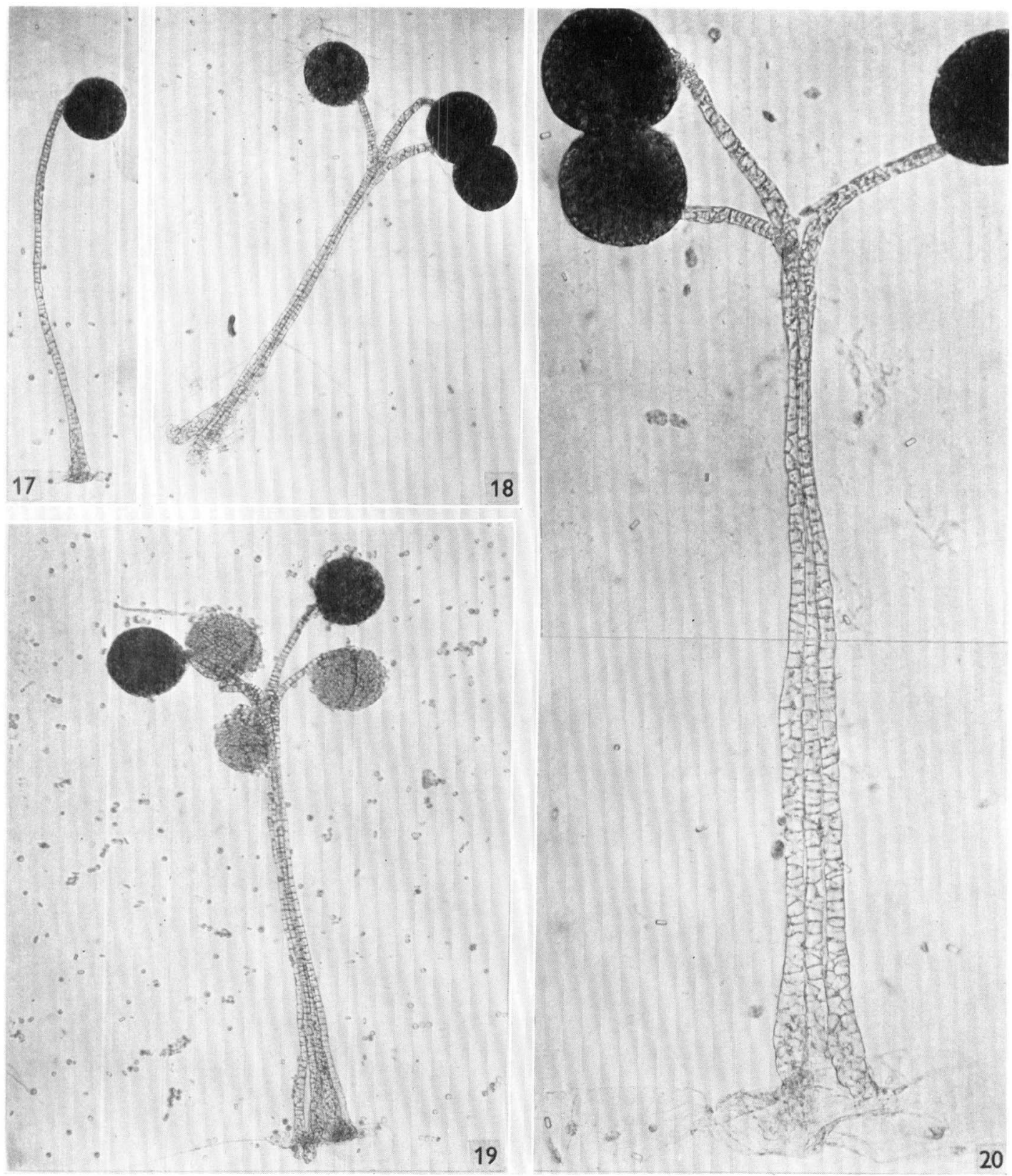

K. B. RAPER-DICTYOSTELIUM POLYGEPLILUM SP.NOv. PLATE :5 